\title{
EXTENSIONS OF FREE GROUPS BY TORSION GROUPS
}

\section{PHILLIP GRIFFITH}

Recently R. G. Swan [5] has proven the following theorem with no commutative assumptions whatsoever: If a torsion free group $G$ contains a free subgroup of finite index, then $G$ is free. Of course, if $G$ is abelian, there is a trivial generalization to the case where $G / F$ is assumed to be bounded with $F$ free (that is, $n(G / F)=0$ where $n \neq 0 \in Z)$. However, in this note we show that Swan's theorem has an even more striking improvement than the one suggested above for $G$ an abelian group. For example, $G / F$ may be a subgroup of a totally projective $p$-group of arbitrary length.

In the ensuing discussion all groups will be abelian and $p$ will be an arbitrary but fixed prime. We denote the group of integers by $Z$ and the group of rational numbers by $Q$. A cotorsion functor $S$ (defined by Nunke in [3]) is one that has a representing sequence $Z \hookrightarrow M \rightarrow H$ where $H$ is torsion such that, for a group $G$, $S G=\operatorname{Image}(\operatorname{Hom}(M, G) \rightarrow \operatorname{Hom}(Z, G) \cong G)$ and if $f: G \rightarrow A$ then $S(f)=f \mid S G$. We further use the notation $S_{p}$ when $H$ is a $p$-group (that is, $S_{p}$ is a $p$-coprimary functor in the sense of Nunke) and call $S_{\boldsymbol{p}}$ reduced if $H$ is reduced. Hill and Megibben [2] call a group $G$ a weak $S$-projective if for any free resolution $F_{0} \mapsto F \rightarrow G$ of $G$ and any group $X$ we have that $\delta_{X}\left(S \operatorname{Hom}\left(F_{0}, X\right)\right)=0$ where

$$
\delta_{X}: \operatorname{Hom}\left(F_{0}, X\right) \rightarrow \operatorname{Ext}(\mathrm{G}, X) .
$$

If $S$ has enough projectives, then a result of Hill and Megibben [2] shows that a $p$-group $G$ is a weak $S$-projective if and only if $G$ is a subgroup of an $S$-projective. Recall that $p G=\{x \in G: x=p y, y \in G\}$ and that for an ordinal $\alpha, p^{\alpha} G=p\left(p^{\alpha-1} G\right)$ if $\alpha-1$ is defined and $p^{\alpha} G=\bigcap_{\beta<\alpha} p^{\beta} G$ if $\alpha$ is a limit ordinal. The functors $p^{\alpha}$ comprise the most well-known and studied examples of the functors described above. Hereafter, we let $S_{p}$ be the class of all $p$-groups that are weak $S_{p}$-projective for some reduced cotorsion functor $S_{p}$. This class contains (in increasing generality) the following classes of $p$-groups:

(1) Arbitrary direct sums of cyclic $p$-groups.

(2) Subgroups of arbitrary direct sums of countable reduced p-groups.

(3) Subgroups of totally projective $p$-groups of arbitrary length. ${ }^{1}$ Before proving the result promised in the introduction, we state

Received by the editors June 3, 1969.

1 A $p$-group $G$ is totally projective if and only if $G / p^{\alpha} G$ is a $p^{\alpha}$-projective for all ordinals $\alpha$. 
a slight generalization of the author's Theorem 2.3 [1]. The proof carries over almost verbatim.

THEOREM. If $\left[M_{i}\right]_{i \in I}$ is a family of groups of finite torsion free rank such that the torsion free subgroups of each $M_{i}$ are free, then each torsion free subgroup of $\sum_{i \in I} M_{i}$ is free.

We remark here that the above theorem can easily be further generalized to the case where each $M_{i}$ is assumed only to have countable torsion free rank.

THEOREM. If $G$ is a torsion free group such that $G$ contains a free subgroup $F$ with $G / F \in S_{p}$, then $G$ is free.

Proof. Let $S_{p}$ denote the reduced cotorsion functor for which $G / F$ is a weak $S_{p}$-projective and let $Z \longmapsto M \rightarrow H$ be its representing sequence. We remark that $Z \subseteq S_{p} M$ and that each torsion free subgroup of $M$ is isomorphic to a subgroup of $Z$. We now form the exact sequence $\sum Z \gg \sum M \rightarrow \sum H$ so that $F \cong \sum Z$. Since $S_{p}$ commutes with sums, there is an imbedding $\psi: \Sigma Z \hookrightarrow S_{p}\left(\sum M\right)$; hence there is a diagram

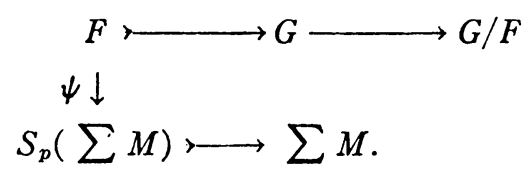

Quoting Hill and Megibben's Theorem 2.2 [2] yields a homomorphism $\phi: G \rightarrow \sum M$ which makes the above diagram commutative. Hence $\operatorname{Ker} \phi \cap F=0$ which implies that $\phi$ is monic since $F$ is necessarily an essential subgroup of $G$. An application of the author's theorem (above) shows that $G$ is free.

Corollary. Let $S_{p}$ be a reduced cotorsion functor with enough projectives. Then each torsion free weak $S_{p}$-projective is free.

Proof. Let $A$ be a torsion free weak $S_{p}$-projective. From Hill and Megibben's Theorems 2.8 and 2.9 [2], we conclude that there is an exact sequence $F>A \rightarrow>B$ where $F$ is free and $B$ is a reduced weak $S_{p}$-projective $p$-group. Thus the theorem shows that $A$ is free.

Hill and Megibben [2] have shown that the class of weak $S_{p}$-projectives is closed under the operations of taking direct sums and of taking subgroups. Hence, one can observe that the assumption on the rank of the groups $M_{i}$ in our first theorem can be dropped if each $M_{i}$ is assumed to be $S_{p}$-projective for some fixed reduced cotorsion functor $S_{p}$. 
CoROLlary. If a torsion free group $G$ contains a free subgroup $F$ such that $G / F$ is contained in a sum of countable reduced p-groups, then $G$ is free.

Our insistance that only one prime be allowed rests upon the fact that there is an immediate counterexample when infinitely many primes are used. For example, for $N$ an infinite subset of the primes there is a noncyclic group $B$ such that $Z \subseteq B \subseteq Q$ and $B / Z \cong \sum_{p \in N} Z(p)$. However, it is clear that the above results remain true when $G / F$ has a finite number of primary components and each of these components is an appropriate weak projective. Although it remains open as to whether $G$ is necessarily free when $G / F$ is only assumed to be a reduced $p$-group, we can make some comment on the general situation. A group $G$ is called $\boldsymbol{\aleph}_{1}$-free if each countable subgroup of $G$ is free and $G$ is called slender (see [4]) if $G$ contains no copy of $Q, \prod_{\mathbb{k}} Z$ or the $q$-adic integers for any prime $q$.

THEOREM. If a torsion free group $G$ is an extension of a free group $F$ by a reduced p-group, then $G$ is $\boldsymbol{\aleph}_{1}$-free and slender.

Proof. If $C$ is a countable subgroup of $G$, then applying the above corollary to the exact sequence $C \cap F \longmapsto C \longrightarrow\{C, F\} / F$ $\subseteq G / F$ shows that $C$ is free. Hence $G$ is $\aleph_{1}$-free. To show that an $\boldsymbol{\aleph}_{1}$-free group is slender, it suffices to show that $\prod_{\aleph_{0}} Z$ cannot be isomorphic to a subgroup of $G$. So suppose that $P \cong \prod \aleph_{0} Z$ is a subgroup of $G$. From the exact sequence $F \cap P \longmapsto P \longrightarrow\{P, F\} / F$ $\subseteq G / F$ and a result of Nunke [4], we obtain that $\{P, F\} / F$ is bounded which further implies the contradictory fact that $P$ is free.

\section{REFERENCES}

1. P. Griffith, A solution to the splitting mixed group problem of Baer, Trans. Amer. Math. Soc. 139 (1969), 261-269.

2. P. Hill and C. Megibben, "On direct sums of countable groups and generalizations," in Études sur les groupes abéliens, B. Charles, Springer-Verlag, Berlin and New York, 1968, pp. 183-206.

3. R. Nunke, Homology and direct sums of countable abelian groups, Math. Z. 101 (1967), 182-212. MR 36 \#1538. \#2508.

4. - Slender groups, Acta. Sci. Math. Szeged 23 (1962), 67-73. MR 26

5. R. Swan, Groups of cohomological dimension one, J. Algebra 12 (1969), 585-610.

University of Chicago 